Modern Physics Letters B

(C) World Scientific Publishing Company

\title{
Spin Berry points as crucial for ultrafast demagnetization
}

\author{
G. P. Zhang* \\ Department of Physics, Indiana State University, Terre Haute, IN 47809, USA \\ *guo-ping.zhang@outlook.com \\ Y. H. Bai \\ Office of Information Technology, Indiana State University, Terre Haute, IN 47809, USA \\ Thomas F. George \\ Departments of Chemistry $\&$ Biochemistry and Physics $\& 3$ Astronomy, University of \\ Missouri-St. Louis, St. Louis, MO 63121, USA \\ Received (March 2, 2021) \\ Revised (Day Month Year)
}

\begin{abstract}
Laser-induced ultrafast demagnetization has puzzled researchers around the world for over two decades. Intrinsic complexity in electronic, magnetic, and phononic subsystems is difficult to understand microscopically. So far it is not possible to explain demagnetization using a single mechanism, which suggests a crucial piece of information still missing. In this paper, we return to a fundamental aspect of physics: spin and its change within each band in the entire Brillouin zone. We employ fcc $\mathrm{Ni}$ as an example and use an extremely dense $\mathbf{k}$ mesh to map out spin changes for every band close to the Fermi level along all the high symmetry lines. To our surprise, spin angular momentum at some special $\mathbf{k}$ points abruptly changes from $\pm \hbar / 2$ to $\mp \hbar / 2$ simply by moving from one crystal momentum point to the next. This explains why intraband transitions, which the spin superdiffusion model is based upon, can induce a sharp spin moment reduction, and why electric current can change spin orientation in spintronics. These special $\mathbf{k}$ points, which are called spin Berry points, are not random and appear when several bands are close to each other, so the Berry potential of spin majority states is different from that of spin minority states. Although within a single band, spin Berry points jump, when we group several neighboring bands together, they form distinctive smooth spin Berry lines. It is the band structure that disrupts those lines. Spin Berry points are crucial to laser-induced ultrafast demagnetization and spintronics.
\end{abstract}

Keywords: Spin Berry phase, ultrafast demagnetization, intraband transition

\section{Introduction}

Laser-induced ultrafast demagnetization on the femtosecond time scale ${ }^{1}$ is interesting because it challenges the current wisdom about the physics of magnetism and ultrafast laser excitation, and because it presents a new opportunity to manipulate spin on an unprecedented time scale. Various mechanisms have been

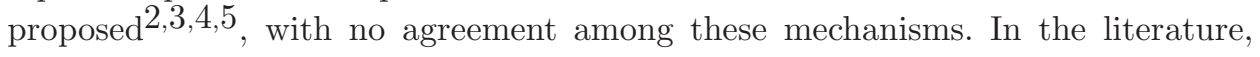


there are at least three pictures, with many questions remaining unanswered. The first is the local picture where the electron spin is quenched on the site. There are several ways as to how such quenching occurs: (i) The electron may pass the angular momentum to the lattice within several hundred femtoseconds. ${ }^{5}$ However, it is well known that phonons only participate in the dynamics on a picosecond time scale, not femtosecond. If phonons are the key to demagnetization, one should expect this fast angular momentum transfer in half-metallic $\mathrm{CrO}_{2} \frac{6}{6}$ and Heusler compounds! Instead, experimentally it is found that the demagnetization time is related to spin polarization. It is likely that this phonon mechanism is only part of the story $[8] 9] 10$

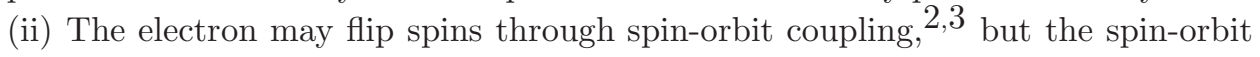
coupling may flip spins in both directions $\left(L^{+} S^{-}+L^{-} S^{+}\right)$, i.e., bidirectional. So there is no guarantee that spin must be reduced. (iii) The electron may transfer the spin angular momentum to orbital. $2\left[11 \mid 12\right.$ This was our original idea, ${ }^{2}$ which was further explored in different settings lately $\frac{11112}{112}$ This mechanism has no issue with the time scale, but is difficult to prove experimentally. Orbital momentum change mainly occurs when the laser field is on! 13 Once the laser field is over, the system evolves within the relative subspaces of spin and orbital. The second difficulty is experimental. In time-resolved x-ray magnetic circular dichroism, there is no experiment that ever reported an increase in orbital momentum, $14 \mid 15$ to the best of our knowledge. On the other hand, the absence of orbital angular momentum increase should not be used as evidence of no angular momentum transfer, because the total angular momentum $\mathbf{J}=\mathbf{L}+\mathbf{S}$ is not a conserved quantity in solids. A reduction in $\mathbf{S}$ does not necessarily lead to an increase in $\mathbf{L}$, because they both can be reduced, but just not at the same rate.

The second is the nonlocal picture where the electron spin is moved out of the region that is excited. This is an itinerant picture where electrons are mobile $\frac{16}{}$ The phenomenological spin superdiffusion mode 4 is based on this picture. The majority spin moves faster than the minority spin, so the deficiency of the majority spin after laser excitation leads to demagnetization. Because the model is phenomenological, the source term that starts the entire demagnetization is lumped into a single term, 17 where no actual laser pulse is present in the theory.

One limitation of the above mechanisms is that they require significant energy absorption. However, experimentally a significant spin reduction is already found 18 even with fluence of around $1 \mathrm{~mJ} / \mathrm{cm}^{2}$. This energy constraint is important as it puts a limit on a theory, so that the incident laser fluence is not used as a fitting parameter. This brings us to the third picture: the spin wave or magnon picture!1913 Here spins are situated at a lattice site and do not move, but their orientations can be changed in space. This directly generates a long-wavelength spin waves across many hundred lattice sites. We recently found that this demagnetization is extremely efficient! 13 To this end, three existing pictures may not be enough to cover all the possible channels to demagnetization. It is imperative to explore new channels.

In this paper, we go back to the basic physics in a ferromagnet. Regardless 
of what the true underlying mechanism is, the basic spin properties remain the same. We take fcc $\mathrm{Ni}$ as an example and examine how spin angular momentum changes in those bands around the Fermi level and across the entire Brillouin zone. To our surprise, by simply moving from one crystal momentum $\mathbf{k}$ point to another within the same band, spin angular momentum undergoes a radical jump from $+\hbar / 2$ to $-\hbar / 2$, or from $-\hbar / 2$ to $+\hbar / 2$. These $\mathbf{k}$ points are not random, and we call them the spin Berry points because they are with the band curvature change. When we group them from neighboring bands, they form smooth lines, namely spin Berry lines. Each high symmetry direction has its unique set of spin Berry points. Along the $\Gamma$-X direction, we have four such spin Berry points, from $\alpha$ to $\gamma$. These Berry points delineate where spins can be flipped through intraband or interband transitions. Microscopically, in presence of spin-orbit coupling, the spin operator does not permute with the total Hamiltonian. The band structure separates spin sectors, so spin angular momentum from the same spin sector appears at two or more different bands. These spin discontinuities are important since they allow the intraband transition to change spin easily. This is the same reason why electric current can change spin orientation in spintronics.

The rest of the paper is arranged as follows. Section 2 is devoted to the interaction between the system and laser field, followed by our theoretical formalism in Sec. 3. Our main results are in Sec. 4, where the band structure and spin angular momentum change along high symmetry directions, and spin Berry points are given. We discuss our results in Sec. 5. Finally, we conclude this paper in Sec. 6 .

\section{Interaction with the laser field}

Laser pulses are an electromagnetic wave. Since it is a transverse wave, the propagation direction is perpendicular to the electric field $\mathbf{E}$ and magnetic field $\mathbf{B}$. To describe the laser interaction with the system, we change the momentum $\mathbf{p}$ to $\mathbf{p}+e \mathbf{A}(\mathbf{r}, t)$, where $\mathbf{A}(\mathbf{r}, t)$ is the laser vector potential. This way the interaction Hamiltonian $H_{I}$ is

$$
H_{I}=\frac{e}{m_{e}}(\mathbf{p} \cdot \mathbf{A}+\mathbf{A} \cdot \mathbf{p})+\frac{e^{2} A^{2}}{2 m_{e}} .
$$

To be definitive, we choose $\mathbf{A}(\mathbf{r}, t)=\mathbf{A}_{0}(t) \mathrm{e}^{i(\mathbf{q} \cdot \mathbf{r}-\omega t)}$, where $\mathbf{q}$ is the light photon wavevector. This is the classical form of the vector potential. In the second quantization, the vector potential is replaced by two photon operators $\left(a^{\dagger}, a\right)$ as $\hat{\mathbf{A}}=\mathbf{A}_{0}(t)\left(a^{\dagger} \mathrm{e}^{i(\mathbf{q} \cdot \mathbf{r}-\omega t)}+a \mathrm{e}^{-i(\mathbf{q} \cdot \mathbf{r}-\omega t)}\right) \underline{20}$, but the spatial and temporal dependences $\mathrm{e}^{i(\mathbf{q} \cdot \mathbf{r}-\omega t)}$ remain unchanged. It must be stated clearly that once $\mathbf{q}$ is chosen, both the electric field and magnetic field directions must be perpendicular to $\mathbf{q}$.

We consider the transition matrix elements of $\mathbf{p} \cdot \mathbf{A}(\mathbf{r}, t)$ between two band states 


$$
\begin{aligned}
& \psi_{m \mathbf{k}_{1}}(\mathbf{r}) \text { and } \psi_{n \mathbf{k}_{2}}(\mathbf{r}), \\
& \qquad \int_{\text {all space }} d \tau \psi_{m \mathbf{k}_{1}}(\mathbf{r})^{*} \mathbf{p} \cdot \mathbf{A}(\mathbf{r}, t) \psi_{n \mathbf{k}_{2}}(\mathbf{r})=\sum_{\mathbf{R}_{l}} e^{i\left(-\mathbf{k}_{1}+\mathbf{q}+\mathbf{k}_{2}\right) \cdot \mathbf{R}_{l}} \\
& \times \int_{\text {u. c. }} d \tau u_{n \mathbf{k}_{1}}(\mathbf{r})^{*} e^{-i \mathbf{k}_{1} \cdot \mathbf{r}} \mathbf{p} \cdot \mathbf{A}_{0}(t) e^{i(\mathbf{q} \cdot \mathbf{r}-\omega t)} u_{m \mathbf{k}_{2}}(\mathbf{r}) e^{i \mathbf{k}_{2} \cdot \mathbf{r}},
\end{aligned}
$$

where $u_{n \mathbf{k}}$ is the periodic part of the Bloch wavefunction $\psi_{n \mathbf{k}}(\mathbf{r})$ and the second integration is over the unit cell only. The summation over the entire lattice site $\mathbf{R}_{l}$ gives $\delta_{-\mathbf{k}_{1}+\mathbf{q}+\mathbf{k}_{2}, 0}$, which guarantees the conservation of the momentum in terms of the crystal momentum. The crystal momentum change is the photon momentum. We make two comments on this. (i) This conservation is restricted to the wave nature of the electron. Since the crystal momentum in solids is not the same as the momentum of the electron, we only can say that there is a small crystal momentum shift during the transition from one band to another. For a laser pulse of wavelength $\lambda,|\mathbf{q}|=2 \pi / \lambda$ should be compared with the reciprocal wavevector $\mathbf{b}$ in the Brillouin zone. In a fcc structure, $|\mathbf{b}|=2 \pi /|\mathbf{a}|$, where $a=|\mathbf{a}|$ is the lattice constant, not to be confused withe creation and annihilation operators above. Suppose $\lambda=800 \mathrm{~nm}$ and $a=3.524 \AA$ (fcc Ni's lattice constant). Then $|\mathbf{q} / \mathbf{b}|=a / \lambda=0.0004405$, which is extremely tiny, so normally one has $\mathbf{k}_{1} \approx \mathbf{k}_{2}$. This favors the vertical interband excitation but ignores intraband transitions in metals. Intraband transitions rely on this tiny change in the crystal momentum in the band states to move from one $\mathbf{k}$ point to another. For instance, if a laser pulse propagates along the $-z$ axis (see Fig. 1(a)), $\mathbf{q}$ is $-q_{z}$, so that the crystal momentum changes along the $k_{z}$ direction.

Another change is in the mechanical momentum $\mathbf{p}+e \mathbf{A}$, where $\mathbf{p}$ is the canonical momentum and $\mathbf{A}$ is the laser field vector potential. This is how we obtain the interaction Hamiltonian with the laser field above. The vector potential also enters intraband transitions through $\mathbf{k} \cdot 16$ If we ignore the spatial dependence of the vector potential and consider a single band, the crystal momentum $\mathbf{k}$ becomes time-dependent and can be approximately written as, 16

$$
\mathbf{k}(t)+e \mathbf{A}(t) / \hbar=\mathbf{k}_{0},
$$

where $\mathbf{k}_{0}$ is the initial crystal momentum. Since $\mathbf{A}$ is perpendicular to $\mathbf{q}$, here the crystal momentum direction is different from the above. If light propagates along the $-z$ axis, only $k_{x}$ and $k_{y}$ are changed (see. Fig. 1(a)). We should point out that Eq. 3 is not generic. This new $\mathbf{k}(t)$ is seemingly similar to $\mathbf{p}+e \mathbf{A}$ divided by $\hbar$, which might prompt one to rewrite the band energy $E_{n}$ as $E_{n}[(\mathbf{p}+e \mathbf{A}) / \hbar]$, which is not strictly correct. ${ }^{21}$ Even if our external laser field is zero (both $\mathbf{E}$ and $\mathbf{B}$ are zero), $\mathbf{A}$ is not necessarily zero and can be a nonzero constant both in space and time. However, if we stick with a single band $E_{n}(\mathbf{k}(t))$, the energy change with time

$$
\frac{\partial E_{n}}{\partial t}=-\frac{e}{\hbar} \nabla_{k} E_{n} \cdot \frac{\partial \mathbf{A}}{\partial t}=\frac{e}{m_{e}} \mathbf{p}_{n n} \cdot \mathbf{E}
$$


where we use $\mathbf{v}_{n n}=\nabla_{k} E_{n} / \hbar=\mathbf{p}_{n n} / m_{e}$. This shows that the band energy changes with the electric field and this change is gauge-invariant, because the band energy is measurable.

Equation 4 is quite insightful. This leads us to investigate how spins are changed under the same laser field excitation.

\section{Theoretical formalism}

We employ the state-of-the-art density functional theory and solve the Kohn-Sham equation, 22 23:24|9

$$
\left[-\frac{\hbar^{2} \nabla^{2}}{2 m_{e}}+V_{n e}+V_{e e}+V_{x c}\right] \psi_{i \mathbf{k}}(\mathbf{r})=E_{i \mathbf{k}} \psi_{i \mathbf{k}}(\mathbf{r}),
$$

where $m_{e}$ is the electron mass, and the terms on the left side are the kinetic energy, nuclear-electron attraction, electron-electron Coulomb repulsion and exchange correlation, respectively. We include the spin-orbit coupling through the secondvariational principle. After we include the spin-orbit coupling, all the states are spin-mixed. Here $\psi_{i \mathbf{k}}(\mathbf{r})$ is the Bloch wavefunction of band $i$ at crystal momentum $\mathbf{k}$, and $E_{i \mathbf{k}}$ is the band energy. We utilize the full-potential augmented plane wave method as implemented in the Wien2k code $\frac{22}{2}$ where within the Muffin-tin sphere atomic basis functions are used, and in the interstitial region a plane wave is used. This method is among the most accurate methods available.

We can define a similar spin change as Eq. 4.

$$
\frac{\partial s_{n \mathbf{k}}^{z}}{\partial t}=\nabla_{k} s_{n \mathbf{k}}^{z} \cdot \frac{e}{\hbar} \frac{\partial \mathbf{A}(t)}{\partial t}=-\frac{e}{\hbar} \nabla_{k} s_{n \mathbf{k}}^{z} \cdot \mathbf{E}(t) .
$$

The unit of spin angular momentum change rate is $\mathrm{Nm}$, a torque that is delivered by an electric field. This equation shows that the spin change rate follows the electric field of the laser pulse, but whether one has a spin reduction or enhancement depends on the spin dispersion with crystal momentum $\mathbf{k}$. Here $s_{n \mathbf{k}}^{z}$ is computed as $\left\langle\psi_{n \mathbf{k}}\left|\hat{s}_{z}\right| \psi_{n \mathbf{k}}\right\rangle$, for band state $\psi_{n \mathbf{k}}$. In the following, we investigate this spin dispersion.

\section{Results}

\subsection{Band structure close to the Fermi level}

We take fcc $\mathrm{Ni}$ as an example. The product of the Muffin-tin radius and plane wave cutoff is 9.5, and we include one local orbital at 0.69 Ryd. As stated above, we include spin-orbit coupling (SOC) at the second variational principle level. This means that we use the spin-polarized band states $\phi_{i \mathbf{k}, \sigma}(\mathbf{r})$, where $\sigma$ denotes the spin orientation, as the basis for the SOC calculation. The final wavefunction is expressed as $\psi_{n \mathbf{k}}(\mathbf{r})=\sum_{i, \sigma} c_{i \mathbf{k}, \sigma}^{n} \phi_{i \mathbf{k}, \sigma}(\mathbf{r})$. This has a benefit that we can extract the spin matrix elements of spin operators $\left(S_{x}, S_{y}\right.$ and $\left.S_{z}\right)$ between bands from $c_{i, \sigma}^{n}$. We express $S_{x}, S_{y}$ and $S_{z}$ in terms of Pauli matrices, $\sigma_{x}, \sigma_{y}$ and $\sigma_{z}$. The k points 

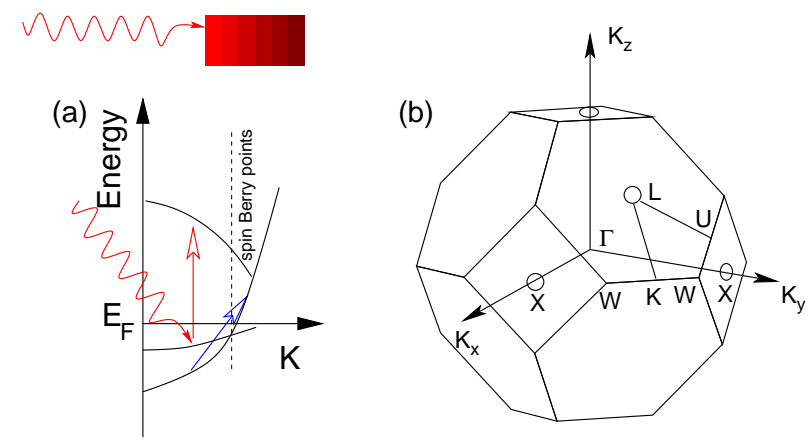

Fig. 1. (a) Two different laser-induced excitations. One is an intraband transition, where electrons only move within the same band. The other is an interband transition between two different bands. What is important to demagnetization is whether these transitions alter spin characters. Top: laser incident on a sample. (b) Three-dimensional Brillouin zone in a fcc lattice. The laser propagates along the $-k_{z}$ axis, with $\mathbf{E}$ and $\mathbf{B}$ in the $k_{x}-k_{y}$ plane. The spin Berry lines are introduced here to understand spin changes during transitions.

along high symmetry lines contain coordinates at $1 / 4$ the reciprocal lattice vector $\mathbf{b}$ (see below), so our number of $\mathbf{k}$ points along $k_{x}, k_{y}$ and $k_{z}$ must be a multiple of 4 . A dense $\mathbf{k}$ mesh grid is necessary. In this work, we employ a mesh of $104 \times 104 \times 104$. There are 73763 irreducible points. There are eight special symmetry lines. Figure 1(b) shows the entire Brillouin zone with the $\Gamma$ point at $(0,0,0)$. All the following coordinates are expressed in the conventional reciprocal lattice vector of a simple cubic, not in a fcc primitive lattice. The length of the reciprocal lattice vector in the conventional lattice is twice that of the primitive lattice.

The $\mathrm{X}$ point is at $(1,0,0)$. There are in total six $\mathrm{X}$ points at $( \pm 1,0,0),(0, \pm 1,0)$, and $(0,0, \pm 1)$, but because $(1,0,0)$ and $(-1,0,0)$ differ by one reciprocal lattice vector, they count as one point. The net number of $\mathrm{X}$ points reduces from 6 to 3 .

The $\mathrm{W}$ points are at $\left(1, \frac{1}{2}, 0\right)$, with the distance from the $\Gamma$ point being $(\sqrt{5} / 2) 2 \pi / a$. They are at the six corners of the hexagons. There are eight hexagons, but two hexagons share two $\mathrm{W}$ points, so there are $6 \times 8 / 2=24 \mathrm{~W}$ points in total. These eight hexagons are enough to create the entire Brillouin. At the middle of two neighboring $\mathrm{W}$ points are $\mathrm{K}$ points. For two $\mathrm{W}$ points at $(1 / 2,1,0)$ and $(1,1 / 2,0), \mathrm{K}$ is at $(3 / 4,3 / 4,0)$. $\mathrm{U}$ is at the middle of another two $\mathrm{W}$ points $(1 / 2,1,0)$ and $(0,1,1 / 2)$, so its coordinate is $(1 / 4,1,1 / 4)$. $\mathrm{U}$ and $\mathrm{K}$ have the same distance to the $\Gamma$ point and are approximately equivalent.

Figure 2 shows the band structure close to the Fermi level (see the horizontal dashed line), with $E_{F}$ at 0.57648 Ryd, which is fully consistent with our prior calculation. 9 One can see the band structure along the W-K and W-U lines is similar. Their bands are away from the Fermi level. Ni has 28 electrons, where 10 electrons are in the core shell, and the remaining 18 electrons are in the valence bands. 10 valence electrons fill the topmost $3 d$ and $4 s p$ bands. Since we are interested in intraband transitions, we only show band states close to the Fermi level. 


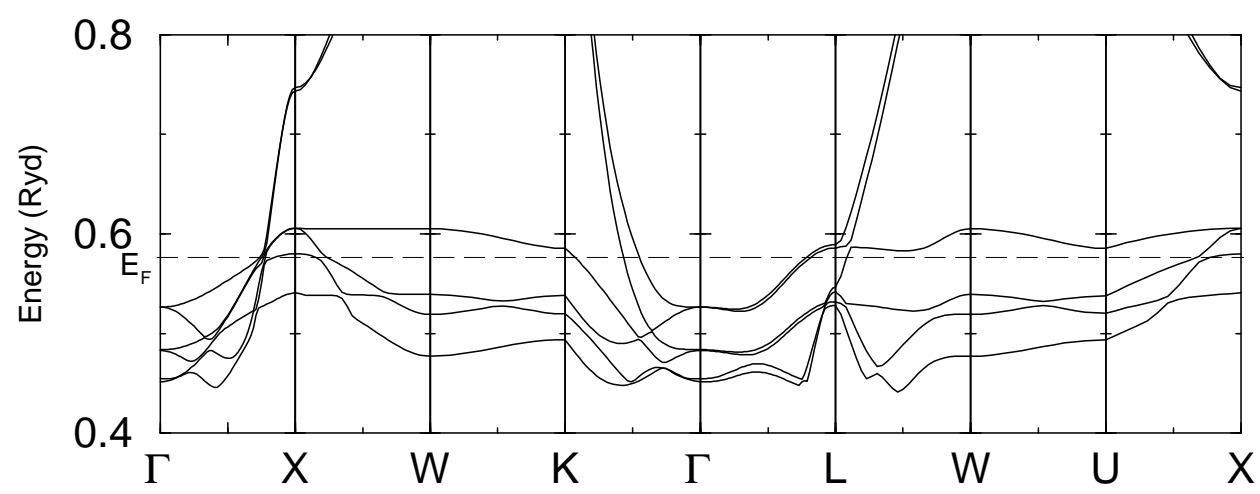

Fig. 2. Band structure close to the Fermi level. The horizontal dashed line denotes the Fermi energy at 0.57642 Ryd.

An important feature among these bands is that they cross each other frequently. For instance, along the $\Gamma$-X direction, $4 s p$ bands, which have a large dispersion, cross the $3 d$ bands which are less dispersive. This crossing has an important consequence on the spin angular momentum. The Fermi level cuts through bands along the $\Gamma$-X, $\mathrm{X}-\mathrm{W}, \mathrm{K}-\Gamma, \Gamma-\mathrm{L}, \mathrm{L}-\mathrm{W}$, and U-X directions. This provides an avenue for intraband transitions, where the electron can move within bands. Investigating how the spin character changes as one moves from one $\mathbf{k}$ point to another is valuable, since it gives us the first hint how laser-driven charge transport leads to magnetization change, as illustrated in Fig. 1(a).

\subsection{Spin change along the $W-K$ and $W-U$ directions}

While the band dispersion in fcc $\mathrm{Ni}$ is well known, little is known as to how each band state changes its spin orientation as we straddle through the Brillouin zone. King et al. ${ }^{25}$ show that in $\mathrm{SrTiO}_{3}$, there is a special spin-orbital texture around the Fermi surface, but $\mathrm{SrTiO}_{3}$ is an insulator, so there are no bands across the Fermi level. For fcc Ni, nothing is known at present. In particular, the Berry phase may change suddenly, so the spin change may show a spike. We decide to choose a simple case where bands in the Brillouin zone do not cross the Fermi level. Naturally, they do not effectively contribute to the spin moment change, but provide a starting point to understand the underlying physics.

As discussed above, band dispersions are similar along the $\mathrm{W}-\mathrm{K}$ and $\mathrm{W}-\mathrm{U}$ directions. We superimpose the spin angular momentum on the bands which are denoted by the empty circles (Fig. 3). The error bars do not represent the error in the band energy; instead they represent spin angular momentum. If the bar is above a circle, this means that the spin angular momentum is positive; below a circle, negative. The length of the bar is proportional to the magnitude of spin $s_{n \mathbf{k}}^{z}$, with the longest bar corresponding to $\hbar / 2$. Figure 3 (a) shows that band 16 has all negative spins, 


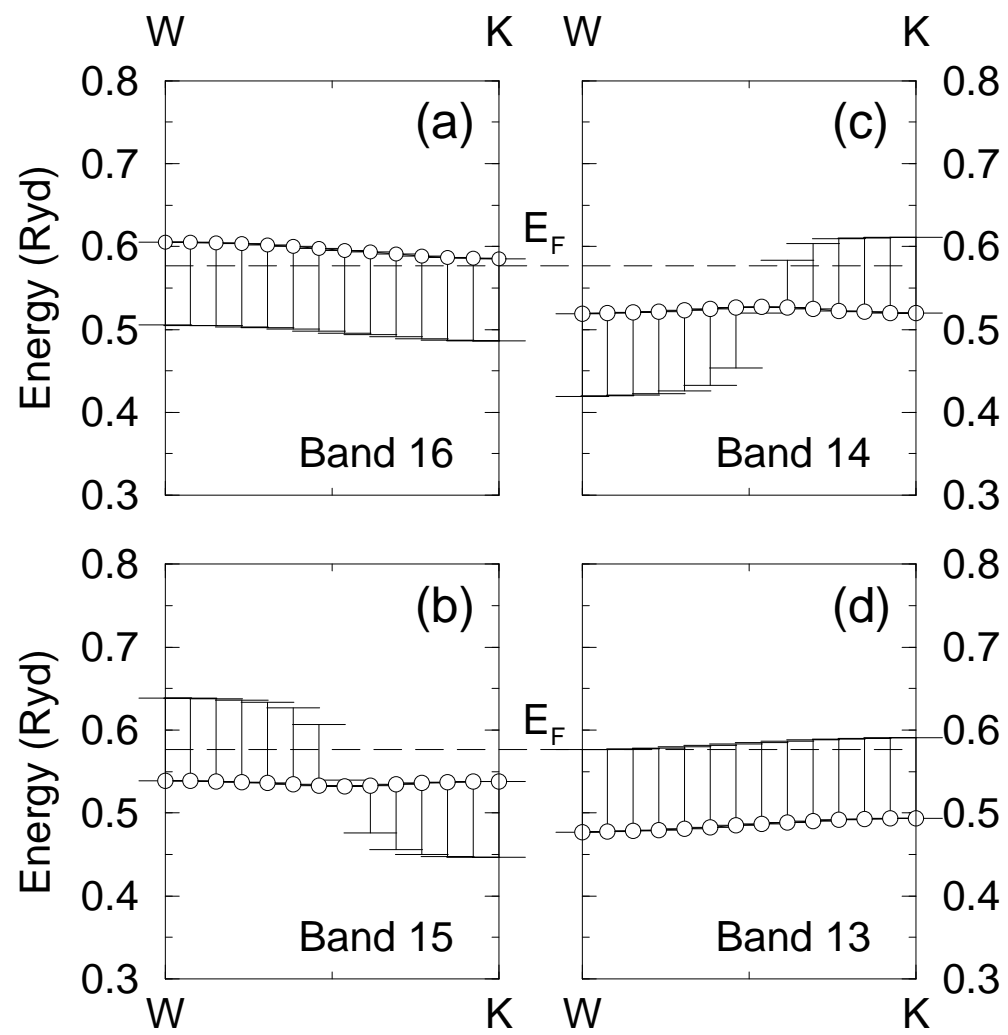

Fig. 3. Spin angular momentum along the W-K direction for four bands that are close to the Fermi surface denoted by horizontal dashed lines. The circles denote the band energy. The error bar denotes the amplitude of the spin angular momentum at each band energy for every band. The longest bar corresponds to $\hbar / 2$. If the bar is below circles, this means that the spin value is negative; if above, positive. Our interest is to see whether spins start to flip. (a) Band 16 and (d) band 13 have a weak spin change from $\mathrm{W}$ to $\mathrm{K}$ since these two bands are away from other bands with little spin mixing. (b) Band 15 and (c) band 14 appear in the same energy window, so they have a strong change in spin angular momentum.

while band 13 (Fig. 3(d)) has positive spins from $\mathrm{W}$ to K. This is understandable. Since they are away from other bands, with or without SOC, there is little interaction to change their spin characters. Because energetically majority and minority bands are shifted with respect to each other due to the exchange interaction, they have different occupations.

By contrast, Figs. 3(b) and 3(c) show that bands 14 and 15 are in the same energy window, so we expect there is a strong interaction between them. Figures 3(b) and 3(c) show that their $s_{n \mathbf{k}}^{z}$ changes sign going from $\mathrm{W}$ to $\mathrm{K}$; and in the middle of the zone, both bands have a very small spin, (see how small those bars are in Figs. 3(b) and 3(c)). We plot the spin change for these two bands in Fig. 4. It is obvious that $s_{n \mathbf{k}}^{z}$ for these two bands is symmetric with respect to 0 (compare 


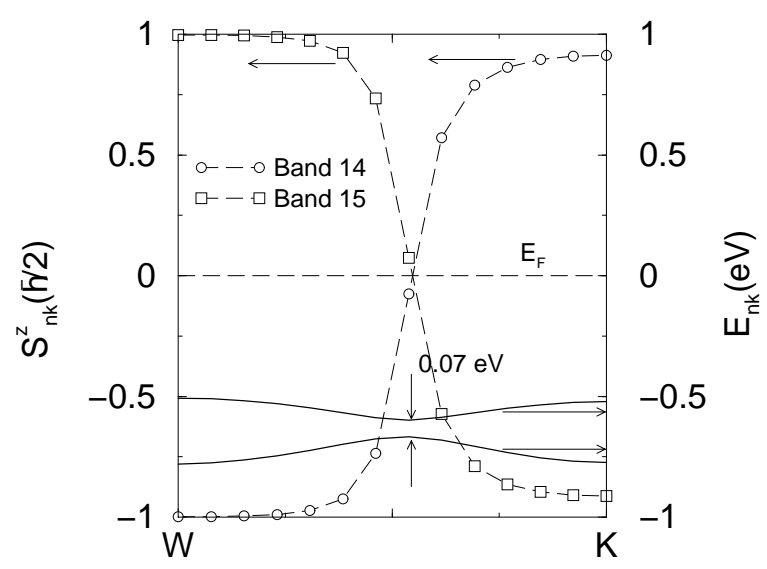

Fig. 4. Spin angular momentum $s_{n \mathbf{k}}^{z}$ in bands 14 and 15 along the W-K direction (use the left scale). The circles refer to spins for band 14 and the boxes for band 15. The Fermi level in this figure is set at zero (horizontal dashed line), and the band energy (two solids lines) is in unit of $\mathrm{eV}$ (right scale).

boxes with circles), and $s_{14, \mathbf{k}}^{z}=-s_{15, \mathbf{k}}^{z}$ along the W-K line. $s_{14, \mathbf{k}}^{z}$ can be fitted to $\tanh \left[\left(-k-k_{0}\right) / c\right]$, where $k_{0}$ and $c$ are two constants. This indicates that the orbital contribution from these two bands is relatively simple, so the spin-flipping term in spin-orbit coupling $\mathbf{L} \cdot \mathbf{S}$ plays a decisive role through

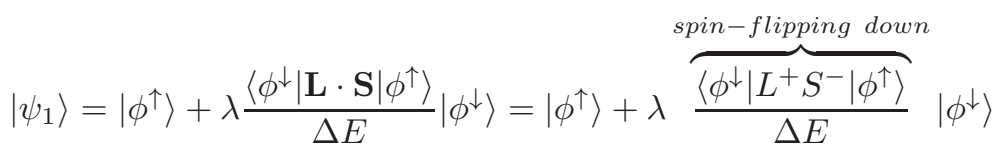

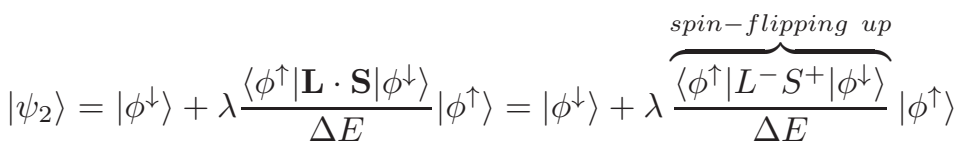

where $\lambda$ is the spin-orbit coupling, $\left|\phi^{\uparrow}\right\rangle$ and $\left|\phi^{\downarrow}\right\rangle$ are the spin-polarized wavefunctions, and $\Delta E$ is the gap between spin majority and minority bands. Two flipping terms depend on $\mathbf{k}$. It should be noted that in this case, angular momentum transfer from spin to orbital is revealed through operators $L^{ \pm}$. It is equally important to point out that one should not expect that the spin and orbital angular momentum changes completely compensate each other, because in solids spherical symmetry is broken. ${ }^{89}$ The minimum gap between these two bands is $0.07 \mathrm{eV}$, which matches the spin-orbit coupling constant of $0.07 \mathrm{eV}$ in our prior model calculation.2 2 These bands are occupied, but if excitation leads to a hole in those bands, a subsequent intraband transition may lead to a spin change. We also compute the spin change along the W-U direction and find the results are similar (not shown). 


\subsection{Spin change along the $X-W$ and $U-X$ directions}

If we look at the Brillouin zone in Fig. 1(b), U, W and X are on the same square. The distance from the $\mathrm{X}$ point at $(0,1,0)$ to $\mathrm{U}$ at $\left(\frac{1}{4}, 1, \frac{1}{4}\right)$ is closer than that to $\mathrm{W}$ at $\left(\frac{1}{2}, 1,0\right)$. What is peculiar about these two directions is that they both have a weakly dispersive unoccupied band, band 16, (see Figs. 5 (a) and $5(\mathrm{e})$ ). This gives us another opportunity to examine the spin change. We see that their spin angular momentum points down and does not change much going from $\mathrm{U}$ to $\mathrm{X}$ and from $\mathrm{X}$ to $\mathrm{W}$. This finding is consistent with our finding above: The less the band interaction is, the weaker the spin change becomes. Figure 5 (b) shows that the first band crosses the Fermi level along the U-X direction, but there is no change in spin angular momentum. This means that even if a laser field is applied along this direction, the intraband transition won't bring in spin change. However, the same band, band 15, along the $\mathrm{X}-\mathrm{W}$ direction has a clear spin change at a point slightly closer to the $\mathrm{W}$ point (highlighted by a box in Fig. 5 (f)), but it does not cross the Fermi level at this box, so intraband transitions do not play a role here. Nevertheless, this is very interesting.

Next we investigate band 14 along the U-X direction (Fig. 5 (c)), where we find a similar spin change in the middle of the zone, highlighted again with a box. If we compare these two boxes in Figs. 罒(f) and 5 (c), we notice that those bands have a common feature where the band curvature changes at those boxes, an infection point. These strange points appear in other bands as well. Figure 5(g) has two such points, while band 13 along both the $\mathrm{U}-\mathrm{X}$ and $\mathrm{X}-\mathrm{W}$ directions have one single point. They always appear at band infection points, which remind us of the Berry phase.26|27

To be definitive, assume that $\psi_{n \mathbf{k}}=e^{i \mathbf{k} \cdot \mathbf{r}}\left(u_{n \mathbf{k}}^{\downarrow}(\mathbf{r}) \beta+u_{n \mathbf{k}}^{\uparrow}(\mathbf{r}) \alpha\right)$, where $u_{n \mathbf{k}}^{\downarrow}(\mathbf{r})$ and $u_{n \mathbf{k}}^{\uparrow}(\mathbf{r})$ are two periodic spatial functions in spin minority and majority channels, and $\alpha$ and $\beta$ are the spin up and spin down states. We can rewrite the expectation value of spin angular momentum as

$$
\left\langle\psi_{n \mathbf{k}}\left|\hat{s}_{z}\right| \psi_{n \mathbf{k}}\right\rangle=\frac{\hbar}{2}\left(\left\langle u_{n \mathbf{k}}^{\uparrow} \mid u_{n \mathbf{k}}^{\uparrow}\right\rangle-\left\langle u_{n \mathbf{k}}^{\downarrow} \mid u_{n \mathbf{k}}^{\downarrow}\right\rangle\right) .
$$

Then the spin gradient $\nabla_{\mathbf{k}} s_{n \mathbf{k}}^{z}$ that enters the spin momentum change in Eq. 6 is

$$
\nabla_{\mathbf{k}} s_{n \mathbf{k}}^{z}=\frac{\hbar}{2}\left(\left\langle\nabla_{\mathbf{k}} u_{n \mathbf{k}}^{\uparrow} \mid u_{n \mathbf{k}}^{\uparrow}\right\rangle+\left\langle u_{n \mathbf{k}}^{\uparrow} \mid \nabla_{\mathbf{k}} u_{n \mathbf{k}}^{\uparrow}\right\rangle\right)-\left(\left\langle\nabla_{\mathbf{k}} u_{n \mathbf{k}}^{\downarrow} \mid u_{n \mathbf{k}}^{\downarrow}\right\rangle+\left\langle u_{n \mathbf{k}}^{\downarrow} \mid \nabla_{\mathbf{k}} u_{n \mathbf{k}}^{\downarrow}\right\rangle\right)
$$

where the two terms are the Berry potentials. $\frac{28}{26}$ The spin gradient is just the difference between the spin majority and minority Berry potentials, or simply the spin Berry potential. To highlight the importance of this Berry phase connection, in Fig. 5 we denote them as spin Berry points. The significance of these points is that before and after those points the spin changes its direction, which is crucial to demagnetization. They occur around those regions where two or more bands cross each other. Each band 13 in Figs. 5 (d) and 5 (h) has a single point. 


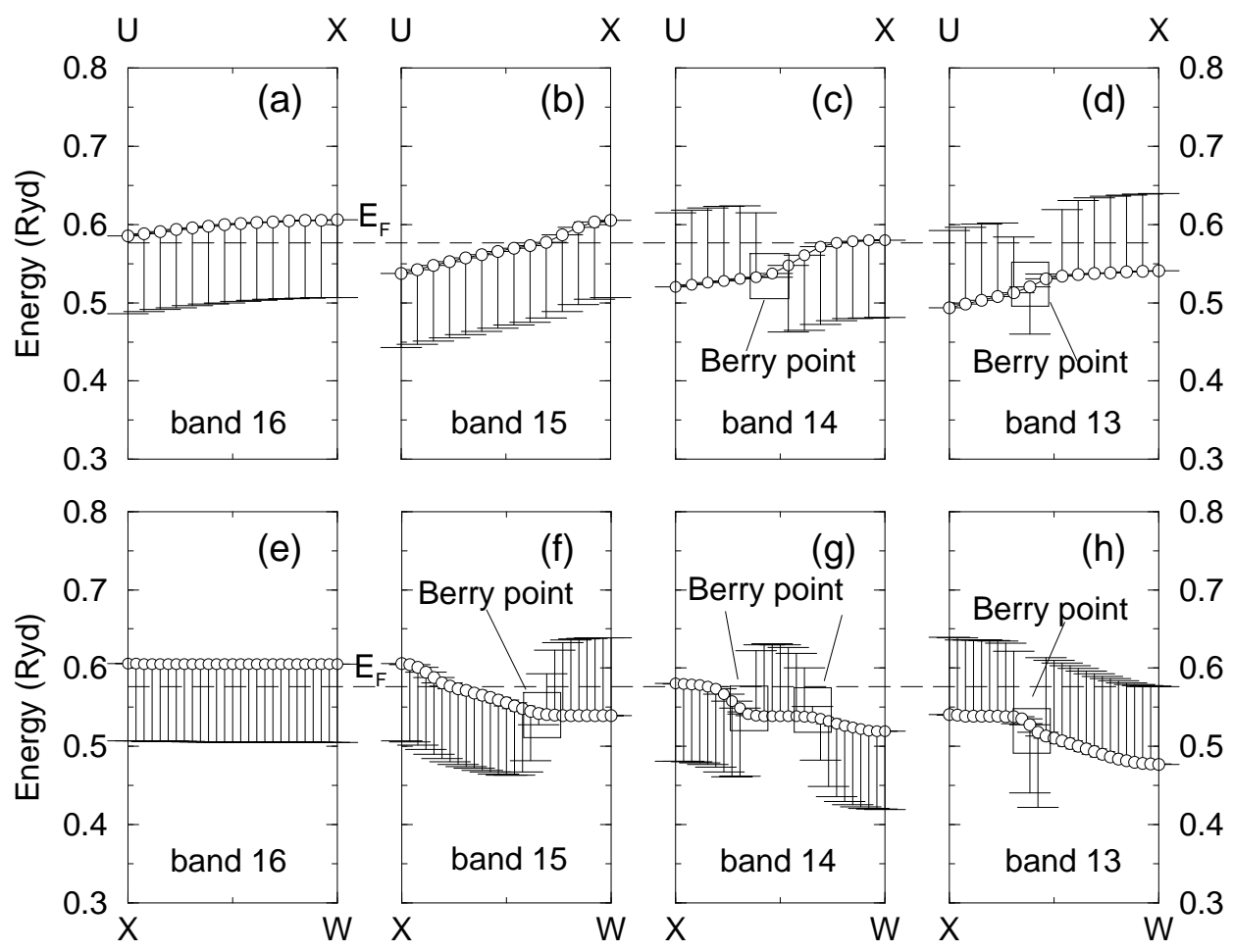

Fig. 5. Spin angular momentum change along the U-X and X-W directions. The longest bar corresponds to $\hbar / 2$. (a), (b), (c) and (d) refer to bands 16, 15, 14 and 13 along the U-X line, respectively. (e), (f), (g) and (h) denote bands from 16, 15, 14 and 13 along the X-W direction. Boxes highlight the spin Berry points. Before and after these points, the spin changes its direction.

Figure 6 shows the spin gradient along the W-K line 1 The unit of the spin angular momentum gradient is $13 \hbar a / \pi \mathrm{b}$ The minimum $\Delta \mathbf{k}$ along the $\mathrm{W}-\mathrm{K}$ line is $\left(\frac{2}{104}, \frac{2}{104}, 0\right) \frac{2 \pi}{a}$, where $a$ is the lattice constant of fcc Ni. Now consider we have a laser pulse with electric field of $0.05 \mathrm{~V} / \AA$, which is commonly used experimentally 18 Figure 6 shows the gradient maximum is $0.653619 \frac{13 \hbar a}{\pi}$. We plug this spin gradient and the laser electric field into Eq. 6. and we find that for every fs, the spin changes by $0.724 \hbar$. This spin change is huge. However, for them to be operative, electrons must be able to move along the band from one $\mathbf{k}$ point to another. This means that the state that an electron moves into must be vacated first. Bands 14 and 15 , as they stand, are not those bands. We will come back to this below with an

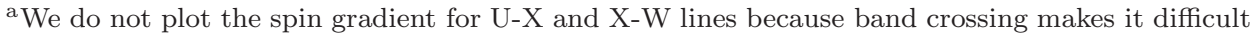
to compute.

bThis unit is calculated as follows. The spin angular momentum has a unit of $\hbar / 2 . \Delta k_{x(y)}=$ $(2 / 104) \frac{2 \pi}{a}$ since our $\mathrm{W}$ point is at $(104,52,0) / 104$, and next point on the line is $(102,54,0) / 104$ until we reach $\mathrm{K}$ at $(78,78,0) / 104$, all in the unit of $\frac{2 \pi}{a}$. 


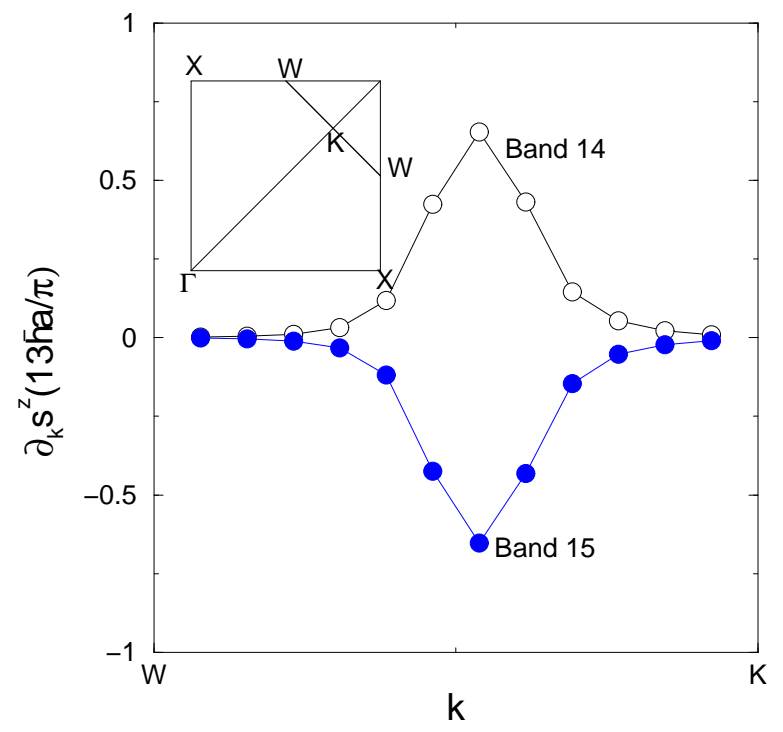

Fig. 6. Spin angular momentum gradient $\nabla_{\mathbf{k}} s_{n \mathbf{k}}^{z}$ along the W-K line for bands 14 (open circles) and 15 (filled circles). $a$ is the lattice constant of fcc Ni. Inset: the square has four special points on the same plane. Note that the unit of the spin gradient is specific to our current $\mathbf{k}$ mesh (see the text for an explanation).

experiment perspective.

\subsection{Spin change along the $\Gamma-L$ and $L$ - $W$ directions}

Whether electron transport affects spin angular momentum depends on whether intraband transitions pass through spin Berry points. Any transition must rigorously obey the Pauli exclusion principle. Consider two electrons in the same band $n$ at two different points, $\mathbf{k}_{1}$ and $\mathbf{k}_{2}$ with occupations $f_{n \mathbf{k}_{1}}$ and $f_{n \mathbf{k}_{2}}$. For such an intraband transition to occur, at least $f_{n \mathbf{k}_{1}}\left(1-f_{n \mathbf{k}_{2}}\right)$ or $f_{n \mathbf{k}_{2}}\left(1-f_{n \mathbf{k}_{1}}\right)$ or both differ from zero. The spin angular momentum change is

$$
\Delta S^{z}=f_{n \mathbf{k}_{1}}\left(1-f_{n \mathbf{k}_{2}}\right)\left(s_{n \mathbf{k}_{2}}^{z}-s_{n \mathbf{k}_{1}}^{z}\right)+f_{n \mathbf{k}_{2}}\left(1-f_{n \mathbf{k}_{1}}\right)\left(s_{n \mathbf{k}_{1}}^{z}-s_{n \mathbf{k}_{2}}^{z}\right),
$$

in the limit that $n \mathbf{k}_{1}$ and $n \mathbf{k}_{2}$ are very close,

$$
\Delta S^{z}=\left[\nabla_{\mathbf{k}} f_{n \mathbf{k}} \cdot \nabla_{\mathbf{k}} s^{z}\right](\Delta k)^{2},
$$

where $\nabla_{\mathbf{k}} s^{z}$ is the spin gradient which connects with the spin Berry potential. Physically, this means that to have a nonzero spin change, the band occupation and spin must be changed simultaneously. $\nabla_{\mathbf{k}} f_{n \mathbf{k}}$ is largest when the state is close to the Fermi level. For this reason, we are mostly interested in those bands close to the Fermi level.

Figures $7(\mathrm{a})$-(d) shows that along $\Gamma$-L direction only bands 18 and 17 cross the Fermi level and are partially occupied, but there is no spin Berry point (see Figs. 


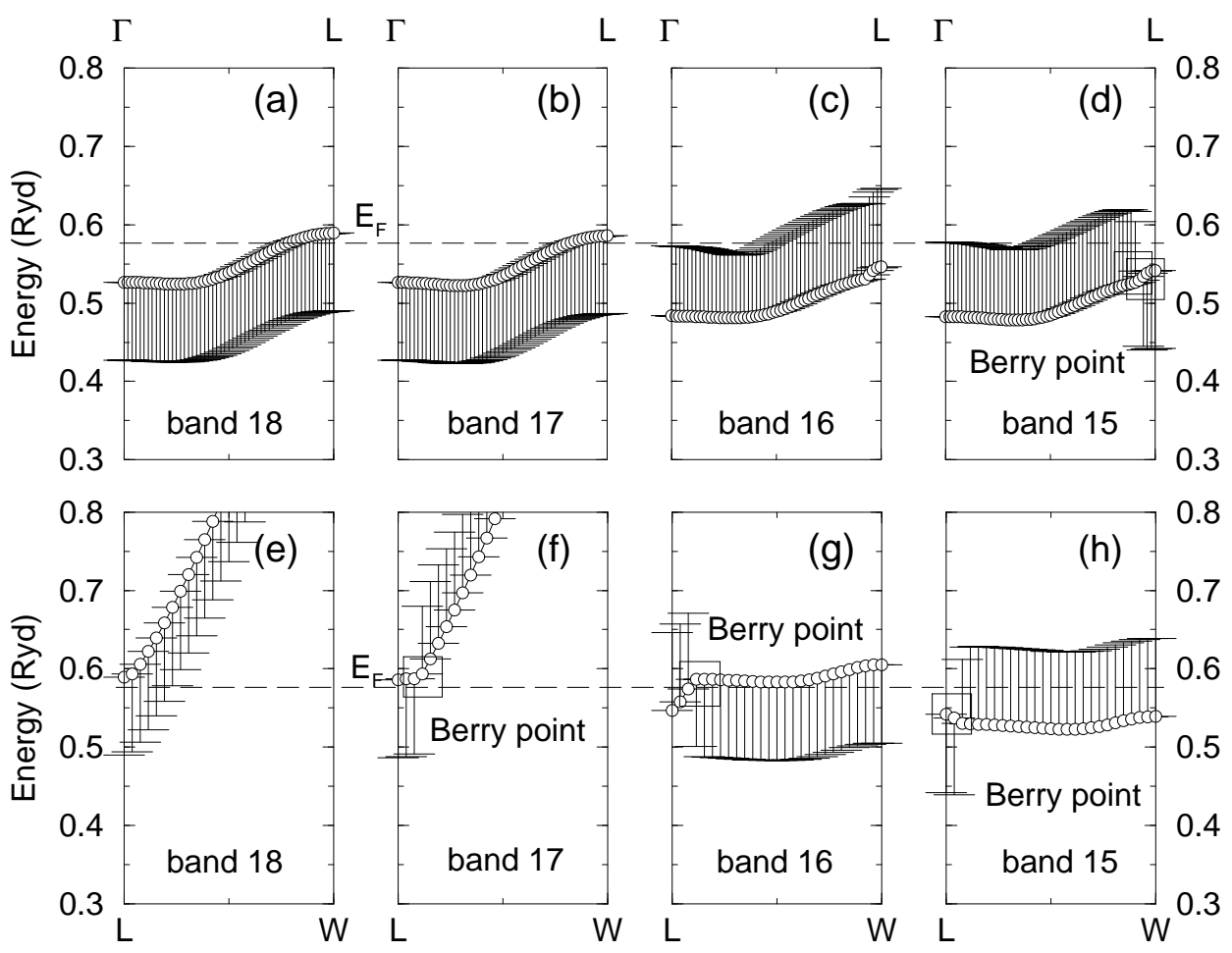

Fig. 7. Band dispersion overlapped with spin angular momentum denoted by the error bar, where the longest bar corresponds to $\hbar / 2$. (a)-(d) shows the spin angular momentum along the $\Gamma-\mathrm{L}$ direction. (e)-(h) shows the results along the L-W direction. Spin Berry points are highlighted with boxes. The Fermi energy is denoted by horizontal dashed lines.

7(a) and 7(b). Bands 16 and 15 are completely filled (see Figs. 7(c) and (d)), but a single spin Berry point is present for band $15 . \mathrm{L}$ is at the center of the hexagon (Fig. 1(b)). Another high symmetry line is the L-W line. Figures 7 (e)-(h) present two different types of bands. Bands 18 and 17 are highly dispersive (Figs. 7(e) and Z(f)), characteristic of $4 s p$ bands. They barely touch the Fermi level. $4 s p$ bands have fewer spin Berry points, but their group velocities are larger. A single spin Berry point in band 17 (Fig. 7 (f)) close to L is directly related to band 16 (Fig. 7(g)). Because of band crossing, a spin Berry point appears (Fig. $7(\mathrm{~g})$ ). Other than this, band 16 has no other Berry point. The situation in band 15 is similar to band 16 and is much less dispersive, characteristic of $3 d$ bands. In addition, it is fully filled as it is below the Fermi level. We also compute the spin change along the $\Gamma-\mathrm{K}$ direction, where the results are similar (not shown). 

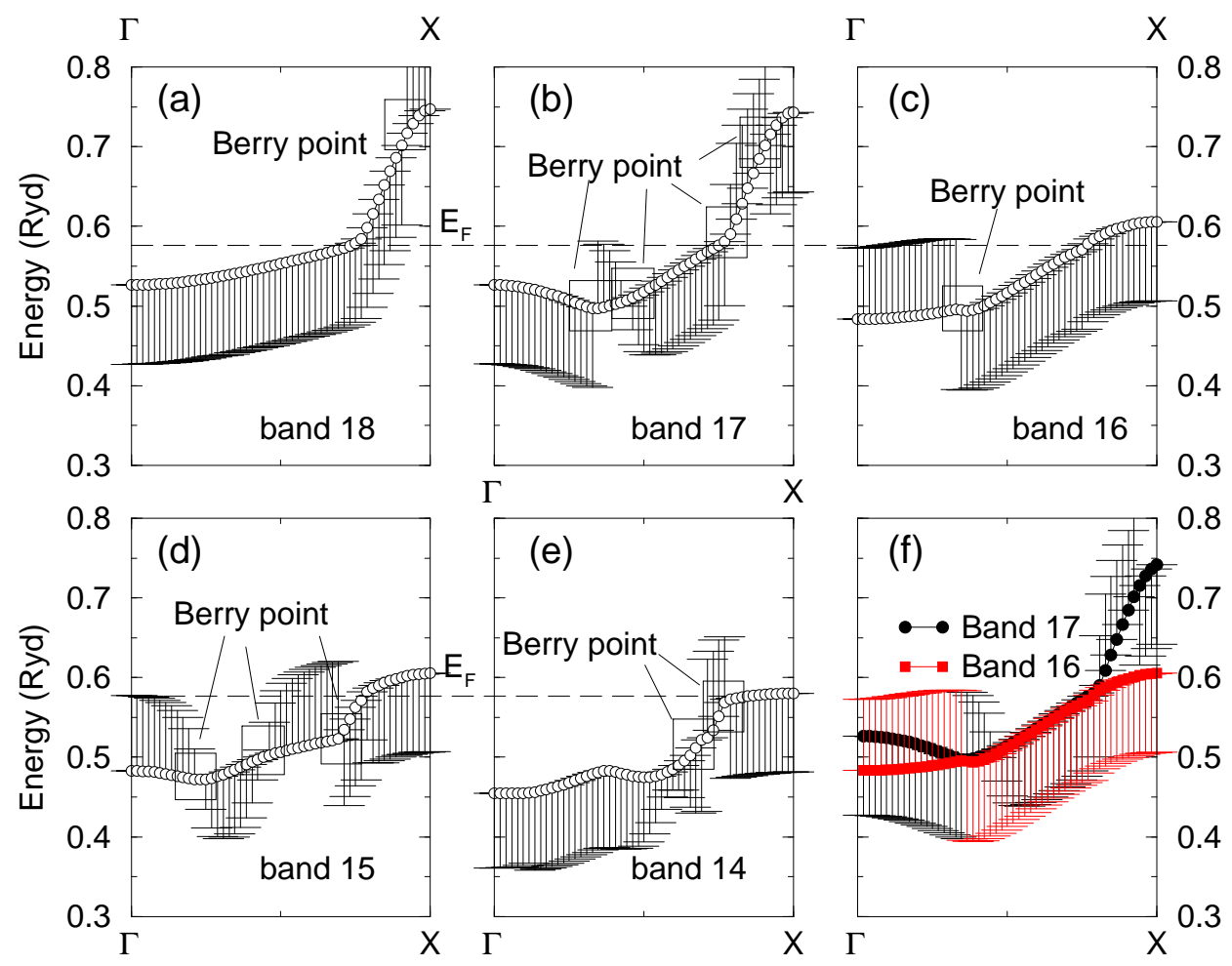

Fig. 8. Spin structure along the $\Gamma-\mathrm{X}$ direction for five bands from band 18 to 14 , where the longest bar corresponds to $\hbar / 2$. (a) Band 18 cuts through the Fermi energy (horizontal dashed line) and has a spin Berry point close to X. (b) Band 17 has four spin Berry points, two below, one at and one above the Fermi level. (c) Band 16 has one point. (d) Band 15 has three. (e) Band 14 has two. (f) Comparison between bands 17 and band 16. Notice that the far left spin Berry point appears at the same location.

\subsection{Spin change along the $\Gamma-X$ direction}

Spin change along the $\Gamma$-X direction is the most complicated and also most interesting one among all the high symmetry lines investigated. While we intend to investigate it first, we immediately realize that it is hard to make sense of it in the beginning. Spin Berry points are present among all the bands at different $\mathbf{k}$ points.

Figure 8 (a) shows that band 18 is partially occupied, and close to the $\mathrm{X}$ point there is a spin Berry point above the Fermi level. Band 17 in Fig. 8(b) has four spin Berry points, the most among all the bands that we investigate. Two points are below the Fermi level. The far left point is linked to the spin Berry point on band 16 (Fig. 8(c)) at the same crystal momentum point. Figure 8(f) compares them in the same graph, where the first $17 \mathrm{k}$ points have spin pointing in opposite directions, but this match ends very quickly since band 15 (Fig. 8 (d)) starts to intrude in the same region of energy. These changes are difficult to track if we do not highlight 
them with spin Berry points. But they are not random. For instance, bands 15 and 14 also interact in the same way. If we compare Figs. $8(\mathrm{~d})$ and (e), the far right spin Berry points are also at the same $\mathbf{k}$. We believe these points are essential to laser-induced intraband demagnetization and spin transport, which deserve extra scrutiny.

\section{Discussion and experimental connections}

Spin Berry points affect not only laser-induced demagnetization but also spin transport across a magnetic sample, because they induce a crucial spin flip when they are driven externally. We need to link them to actual experiments as how to probe them. But at first, we must establish that these points are not random. Spin Berry points up to now are presented band by band, so we can see them clearly as to how they change across the Brillouin zone. However, this does not give the entire picture.

Figure 9 shows a different picture along the $\Gamma$-X line, where we increase one band at a time from Figs. 9(a) to 9(e), where bands 18, 17, 16, 15 and 14 are denoted by circles, boxes, diamonds, up triangles and down triangles, respectively. Three vertical dashed lines $\alpha, \beta, \beta^{\prime}$, and $\gamma$ separate four spin Berry zones. Figure 9(a) shows that the spin angular momentum stays at $-\hbar / 2$ (see circles) but then when it hits the $\gamma$ line, it jumps from $-\hbar / 2$ to $\hbar / 2$. It is difficult to understand why spin can have such a radical change to flip spins suddenly. However, if we add band 17, it immediately patches this spin jump at the $\gamma$ line with more points (see boxes in Fig. 9 (b)), so the jump is not steep anymore. This indicates that the spin angular momentum change is shared by two bands. In other words, in the band structure, we consider bands 18 and 17 are two different energy bands, but in the spin space, some of them belong to the same spin band. Microscopically, spin-orbit coupling does not permute with the original Hamiltonian and creates two new sets of bands from two spin-polarized bands, but the spin-part of the wavefunctions is still correlated. This finding is interesting, so we decide to include more bands.

We notice that Fig. 9(b) shows that band 17 introduces a new spin jump at the $\alpha$ line. Figure 9(c) shows that at the $\alpha$ line the inclusion of band 16 complements the initial jump left behind by band 17 . The diamonds before the $\alpha$ line smoothly merge into boxes, and boxes further merge into circles at the $\gamma$ line, completing a single line, called, the spin Berry line. The boxes before the $\alpha$ line smoothly merge into diamonds along the way to the $\mathrm{X}$ point, completing another spin Berry line. Band 15 in Fig. 9(d) introduces a new line from $\Gamma$ to a point close to the $\beta$ line (see up triangles), and then suddenly drops to $-\hbar / 2$. This sudden jump is removed by band 14 (down triangles in Fig. 9(e)) at the same location. Band 14 has a peak between the $\alpha$ and $\beta$ lines. Along the $\Gamma-\mathrm{X}$ line, we can at least identify five spin Berry lines - line 1: $\Gamma-\diamond-\alpha-\square-\gamma-\bigcirc-\mathrm{X}$, line 2: $\Gamma-\bigcirc-\alpha-\bigcirc-\gamma-\square-\mathrm{X}$, line 3: $\Gamma-\square-\alpha-\diamond-\gamma-\diamond-\mathrm{X}$; line $4: \Gamma-\triangle-\beta^{\prime}-\nabla-\beta-\triangle-\mathrm{X}$, line 5 : $\Gamma-\nabla-\beta^{\prime}-\triangle-\beta-\nabla-\mathrm{X}$. 


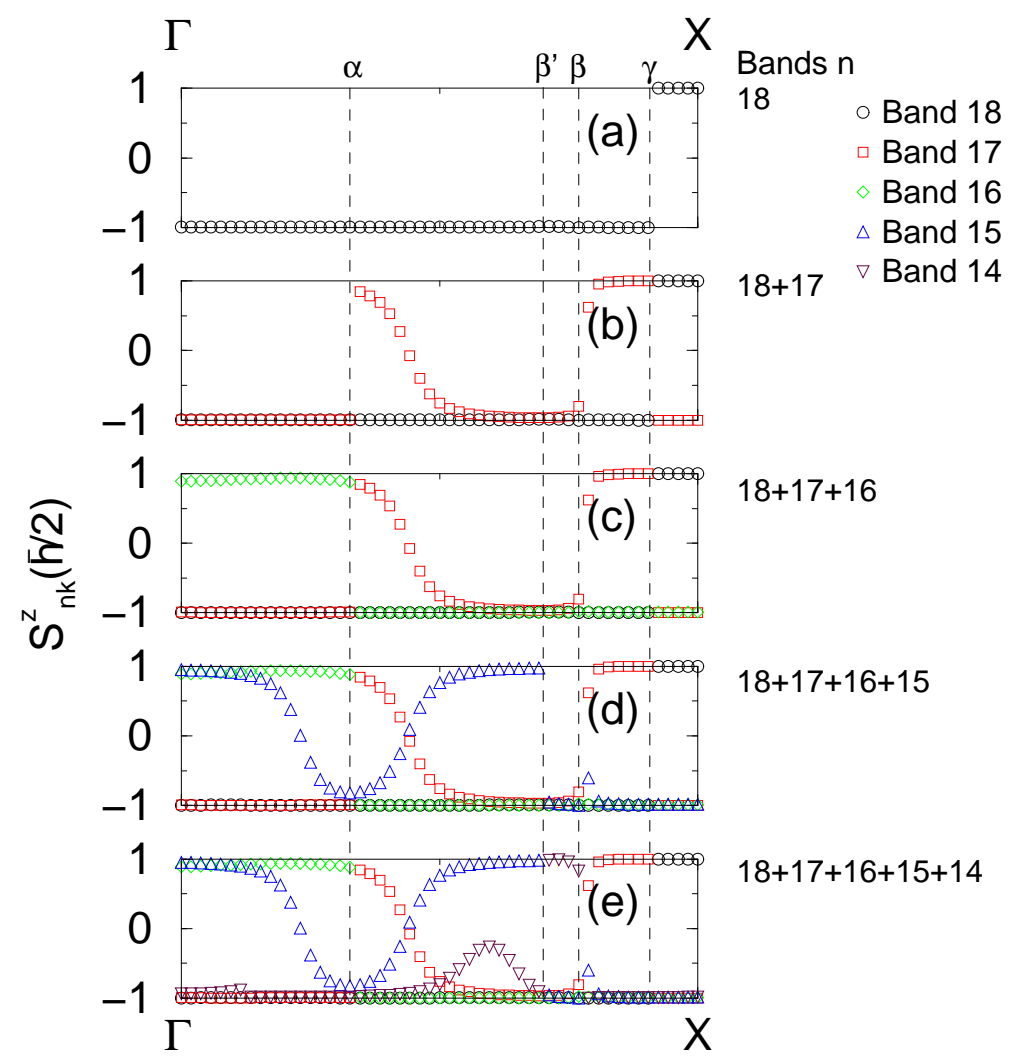

Fig. 9. Establishing spin Berry lines by gradually including more bands. Three vertical dashed lines denote where spins should be connected. (a) Only band 18 is present (circles). There is a sharp step jump at the $\gamma$ line. (b) Inclusion of band 17 softens the jump in spin by attaching more points at the $\gamma$ line (see boxes), but generates a new jump at the $\alpha$ line (boxes). (c) Band 16 directly attaches the spin points at the $\alpha$ line (diamonds), so the jump does not appear anymore. (d) Band 15 contributes a new spin curve (up triangles), but introduces a new jump at the $\beta$ line. (e) Inclusion of band 14 removes the sharp jump at $\beta$ line, in the same way as above. Spin Berry points are linked among different bands, and are in fact spin Berry lines. There are five lines - line 1: $\Gamma-\diamond-\alpha-\square-\gamma-\bigcirc-\mathrm{X}$, line 2: $\Gamma-\bigcirc-\alpha-\bigcirc-\gamma-\square-\mathrm{X}$, line 3: $\Gamma-\square-\alpha-\diamond-\gamma-\diamond-\mathrm{X}$; line 4: $\Gamma-\triangle-\beta^{\prime}-\nabla-\beta-\triangle-\mathrm{X}$, line $5: \Gamma-\nabla-\beta^{\prime}-\triangle-\beta-\nabla-\mathrm{X}$.

It is important to make connections with experiments. Detection of Berry's phase in BiTe ${ }^{29}$ and superconducting charge pump 30 was reported. Our prediction does not require those exotic phases. 31 There are two types of bands that experimentally one can probe. One type is where those bands directly cross the Fermi level. They are partially occupied. Suppose that one can apply a voltage bias along the $\Gamma$-X direction for bands 14 and 17. Spins in this band can be switched from one direction to another. If one employs a laser pulse, a similar effect can be obtained as well. Optically, these are intraband transitions, where a low energy photon, maybe in the infrared region, drives electrons across the Fermi level. This 
is probably the reason behind spin flipping observed experimentally $32 \sqrt[33]{3}$ If this happens between two materials with different spin polarization, spin injection can be achieved.

The second type is related to interband transition. As seen in several figures, there are fewer directions along which band states have spin flipping. But in those bands that are away below the Fermi level, multiple spin Berry points are noticed. These bands are occupied, so without any excitation, electrons cannot move. A possibility arises that if electrons are first excited to an excited state, then a subsequent intraband transition may lead to a strong spin moment change in the valence band. Time- and spin-resolved photoemission has the potential to resolve spin change along different crystal momentum directions, and finally identify those spin Berry points and Berry lines.

\section{Conclusion}

Different from many prior studies, we focus on how the spin angular momentum changes with the crystal momentum for those bands that are close to the Fermi surface. We have employed fcc $\mathrm{Ni}$ as an example, and we find that the spin dispersion with the crystal momentum has abrupt jumps from $\pm \hbar / 2$ to $\mp \hbar / 2$, very unusual in the first glance. We notice those jumps only appear when two or more bands are close by. These special crystal momentum points are called spin Berry points, where the spin Berry potential for the spin majority and spin minority bands is different. When we group several bands together, we find these spin Berry points in fact form a smooth spin Berry line, and there are many of these lines present along a particular direction. Because these bands participate in intraband transitions and spin transport, the information that we find here is very important to both laser-induced ultrafast demagnetization and spintronics. We expect that time- and spin-resolved photoemission is able to detect them.

This work was supported by the U.S. Department of Energy under Contract No. DE-FG02-06ER46304. We acknowledge part of the work as done on Indiana State University's high-performance computers. This research used resources of the National Energy Research Scientific Computing Center, which is supported by the Office of Science of the U.S. Department of Energy under Contract No. DE-AC0205CH11231. Calculations used resources of the Argonne Leadership Computing Facility at Argonne National Laboratory, which is supported by the Office of Science of the U.S. Department of Energy under Contract No. DE-AC02-06CH11357.

*guo-ping.zhang@outlook.com. https://orcid.org/0000-0002-1792-2701

\section{References}

1. E. Beaurepaire, J. C. Merle, A. Daunois, and J.-Y. Bigot, Ultrafast spin dynamics in ferromagnetic nickel, Phys. Rev. Lett. 76, 4250 (1996).

2. G. P. Zhang and W. Hübner, Laser-induced ultrafast demagnetization in ferromagnetic metals, Phys. Rev. Lett. 85, 3025 (2000). 
3. B. Koopmans, G. Malinowski, F. Dalla Longa, D. Steiauf, M. Fähnle, T. Roth, M. Cinchetti, and M. Aeschlimann, Explaining the paradoxical diversity of ultrafast laserinduced demagnetization, Nat. Mater. 9, 259 (2010).

4. M. Battiato, K. Carva, and P. M. Oppeneer, Superdiffusive spin transport as a mechanism of ultrafast demagnetization, Phys. Rev. Lett. 105, 027203 (2010).

5. C. Dornes et al., The ultrafast Einstein-de Haas effect, Nature 565, 209 (2019).

6. Q. Zhang, A. V. Nurmikko, G. X. Miao, G. Xiao, and A. Gupta, Ultrafast spindynamics in half-metallic $\mathrm{CrO}_{2}$ thin films, Phys. Rev. B 74, 064414 (2006).

7. G. M. Müller, J. Walowski, M. Djordjevic, G.-X. Miao, A. Gupta, A. V. Ramos, K. Gehrke, V. Moshnyaga, K. Samwer, J. Schmalhorst, A. Thomas, A. Hütten, G. Reiss, J. S. Moodera, and M. Münzenberg, Spin polarization in half-metals probed by femtosecond spin excitation, Nat. Mater. 8, 56 (2009).

8. G. P. Zhang and T. F. George, Total angular momentum conservation in laser-induced femtosecond magnetism, Phys. Rev. B 78, 052407 (2008).

9. G. P. Zhang, Y. H. Bai, and T. F. George, Energy- and crystal momentum-resolved study of laser-induced femtosecond magnetism, Phys. Rev. B 80, 214415 (2009).

10. C. Stamm, N. Pontius, T. Kachel, M. Wietstruk, and H. A. Dürr, Femtosecond x-ray absorption spectroscopy of spin and orbital angular momentum in photoexcited $\mathrm{Ni}$ films during ultrafast demagnetization, Phys. Rev. B 81, 104425 (2010).

11. W. Töws and G. M. Pastor, Many-body theory of ultrafast demagnetization and angular momentu transfer in ferromagnetic transition metals, Phys. Rev. Lett. 115, 217204 (2015).

12. J. K. Dewhurst, S. Shallcross, P. Elliott, S. Eisebitt, C. v. Korf Schmising, and S. Sharma, Where does the spin angular momentum go in laser induced demagnetisation?, arXiv: $2012.03657 \mathrm{v} 1$.

13. G. P. Zhang, M. Murakami, Y. H. Bai, T. F. George, and X. S. Wu, Spin-orbit torquemediated spin-wave excitation as an alternative paradigm for femtomagnetism, J. Appl. Phys. 126, 103906 (2019).

14. C. Boeglin, E. Beaurepaire, V. Halte, V. Lopez-Flores, C. Stamm, N. Pontius, H. A. Dürr, and J.-Y. Bigot, Distinguishing the ultrafast dynamics of spin and orbital moments in solids, Nature 465, 458 (2010).

15. N. Bergeard, V. Lopez-Flores, V. Halte, M. Hehn, C. Stamm, N. Pontius, E. Beaurepaire, and C. Boeglin, Ultrafast angular momentum transfer in multisublattice ferrimagnets, Nat. Commun. 5, 3466 (2014).

16. G. P. Zhang, Y. H. Bai, T. Jenkins, and T. F. George, Laser-induced ultrafast transport and demagnetization at the earliest time: First-principles and real-time investigation, J. Phys.: Condens. Matter 30, 465801 (2018).

17. M. Battiato, K. Carva, and P. M. Oppeneer, Theory of laser-induced ultrafast superdiffusive spin transport in layered heterostructures, Phys. Rev. B 86, 024404 (2012).

18. M. S. Si and G. P. Zhang, Resolving photon-shortage mystery in femtosecond magnetism, J. Phys.: Condens. Matter 22, 076005 (2010).

19. E. Turgut, D. Zusin, D. Legut, K. Carva, R. Knut, J. M. Shaw, C. Chen, Z. Tao, H. T. Nembach, T. J. Silva, S. Mathias, M. Aeschlimann, P. M. Oppeneer, H. C. Kapteyn, M. M. Murnane, and P. Grychtol, Stoner versus Heisenberg: Ultrafast exchange reduction and magnon generation during laser-induced demagnetization, Phys. Rev. B 94, 220408 (2016).

20. M. O. Scully and M. S. Zubairy, Quantum Optics, Cambridge University Press, Cambridge (2006).

21. J. Callaway, Quantum Theory of the Solid State, Academic Press, Inc., New York (1974). 
22. P. Blaha, K. Schwarz, G. K. H. Madsen, D. Kvasnicka, and J. Luitz, WIEN2k, An Augmented Plane Wave + Local Orbitals Program for Calculating Crystal Properties (Karlheinz Schwarz, Techn. Universität Wien, Austria, 2001).

23. P. Blaha, K. Schwarz, F. Tran, R. Laskowski, G. K. H. Madsen and L. D. Marks, WIEN2k: An APW+lo program for calculating the properties o f solids, J. Chem. Phys. 152, 074101 (2020).

24. G. P. Zhang, W. Hübner, G. Lefkidis, Y. Bai, and T. F. George, Paradigm of the time-resolved magneto-optical Kerr effect for femtosecond magnetism, Nat. Phys. 5, 499 (2009).

25. P. D. C. King, S. M. Walker, A. Tamai, A. de la Torre, T. Eknapakul, P. Buaphet, S.-K. Mo, W. Meevasana, M.S. Bahramy, and F. Baumberger, Quasiparticle dynamics and spin-orbital texture of the $\mathrm{SrTiO}_{3}$ two-dimensional electron gas, Nat. Commun. 5, 3414 (2014).

26. M. V. Berry, Quantal phase factors accompanying adiabatic changes, Proc. R. Soc. Lond. A 392, 45 (1984).

27. J. W. Zwanziger, M. Koenig, and A. Pines, Berry's phase, Ann. Rev. Phys. Chem. 41, 601 (1990).

28. D. Vanderbilt, Berry Phases in Electronic Structure Theory, Cambridge University Press, Cambridge (2018).

29. H. Murakawa, M. S. Bahramy, M. Tokunaga, Y. Kohama, C. Bell, Y. Kaneko, N. Nagaosa, H. Y. Hwang, Y. Tokura1, Detection of Berry's phase in a bulk rashba semiconductor, Science 342, 1490 (2013).

30. M. Möttönen, J. J. Vartiainen, and J. P. Pekola, Experimental determination of the Berry phase in a superconducting charge pump, Phys. Rev. Lett. 100, 177201 (2008).

31. V. Adak, K. Roychowdhury, and S. Das, Spin Berry phase in a helical edge state: $\mathrm{S}_{z}$ nonconservation and transport signatures, Phys. Rev. B 102, 035423 (2020).

32. E. Turgut, C. La-o-vorakiat, J. M. Shaw, P. Grychtol, H. T. Nembach, D. Rudolf, R. Adam, M. Aeschlimann, C. M. Schneider, T. J. Silva, M. M. Murnane, H. C. Kapteyn, and S. Mathias, Controlling the competition between optically induced ultrafast spinflip scattering and spin transport in magnetic multilayers, Phys. Rev. Lett. 110, 197201 (2013).

33. S. Eich, M. Plötzing, M. Rollinger, S. Emmerich, R. Adam, C. Chen, H. C. Kapteyn, M. M. Murnane, L. Plucinski, D. Steil, B. Stadtmüller, M. Cinchetti, M. Aeschlimann, C. M. Schneider and S. Mathias, Band structure evolution during the ultrafast ferromagnetic-paramagnetic phase transition in cobalt, Sci. Adv. 3, e1602094 (2017). 